\title{
THE LANDAU THEORY OF PHASE TRANSITIONS
}


This page is intentionally left blank 


\section{THE LANDAU THEORY OF PHASE TRANSITIONS}

Application to Structural, Incommensurate, Magnetic, and Liquid Crystal Systems.

\section{Jean-Claude TOLÉDANO}

Centre National d'Etudes des

Télécommunications

FRANCE

\section{Pierre}

\section{TOLÉDANO}

University of Amiens

FRANCE 
Published by

World Scientific Publishing Co. Pte. Ltd.

P.O. Box 128, Farrer Road, Singapore 9128

U.S.A. office: World Scientific Publishing Co., Inc. 687 Hartwell Street, Teaneck NJ 07666, USA

Library of Congress Cataloging-in-Publication data is available.

\section{THE LANDAU THEORY OF PHASE TRANSITIONS}

Copyright $\odot 1987$ by World Scientific Publishing Co Pte Ltd.

All rights reserved. This book, or parts thereof, may not be reproduced in any form or by any means, electronic or mechanical, including photo. copying, recording or any information storage and retrieval system now known or to be invented, without written permission from the Publisher.

$\begin{array}{ll}\text { ISBN } & 9971-50-025-6 \\ & 9971-50-026-4(\mathrm{pbk})\end{array}$

Printed in Singapore by Kim Hup Lee Printing Co. Pte. Ltd. 
A NOS PARENTS 
This page is intentionally left blank 
FOREWORD

Why a book on Landau's theory of phase transitions ? To many physicists working in the fleld of phase transitions, this question will appear as doubly relevant. Indeed, why describe in detall the foundations and consequences of a theory whose basic hypothesis (the absence of singularity in the transition free-energy), and whose essential physical result (the specification of a critical behaviour) have been known for 40 years to be questionable ? Why, on the other hand, restrict to this phenomenological approach, at a time when microscopic models can be handled by various theoretical and numerical methods, and provide a "royal way" to the investigation of phase transitions?

The existence of a satisfactory answer to these questions is attested by the fact that, ever since its formulation, Landau's theory has been used without interruption as the theoretical background of many studies of systems undergoing phase transitions. More strikingly, it is in the last 15 years, after the advent of the modern statistical theory of critical phenomena, that the utility of Landau's theory has been demonstrated most clearly, when tt has been applied to the lntricate patterns of transitions observed in the structural, magnetic, and 11quidcrystalline systems, and more recently, to the investigation of the stabilitles and properties of the incommensurate phases, and of the Icosahedral quasi-crystalline phases.

There are several reasons to this persistent use of Landau's theory. A first set of reasons is related to the fact that the objected lack of validity of the basic assumptions and results of Landau's theory is not of a clearcut nature. Thus, the symetry aspects which constitute an important part of the theory, are rigorous. Besides, there are classes of systems, governed by long range Interactions (e.g. elastic interactions) for which the critical behaviour is expected to be correctly described by Landau's theory. More significantly, the temperature range adjacent to the transitions, in which the behaviour of a system is dominated by the fluctuations (and in which, accordingly Landau's theory falls), usually constitutes a small fraction of the temperature range of experimental interest. In most of the latter range, the Landau theory is an adequate tool to investigate the physical behaviour of the system. 
Another Important set of reasons pertains to the possibllity of manipulating, through a mathematically simple and flexible theory, the complicated degrees of freedom which describe the states of real systems (e.g. sets of collective atomic displacements, or intricate spin configurations). Likewise, one has the possibility of relating simply to eachother a variety of physical properties (mechanical, optical, lattice-dynamical, structural,...) whose microscopic description would be very difficult.

The mathematical simplicfty of the theory is a consequence of the clever manner by which Landau defines the order-parameter through the substitution of a small set of scalar (spatially uniform) quantities, to a set of functions having rapid variations at the atomic level. This substitution which appears as a "trick" of mere mathematical convenience, has the important consequence of permitting the description of the evolution of a complex spatial configuration of particles by means of an ordinary polynomial expansion. This trick also gives to the order-parameter a duality of meanings. As a spatlally uniform quantity (or a smoothly varying one, in certain cases) it can be considered as of macroscopic nature. On the other hand, the functions 1t substitutes are clearly of microscopic nature. At choice, one can put the accent on one interpretation or the other (e.g. on the dielectric polarization of a ferroelectrlc crystal, or on the structural changes and lattice dynamical mode related to the polarization).

The flexiblity of the theory resides in its modular character. Aside from the primary order-parameter, additional degrees of freedom can be incorporated in the theory as measure as the acquisition of the experimental data requires lnterpreting a larger set of results. For instance, in the study of a crystalline transition, once the primary order-parameter is given a sense in terms of atomic displacements, one can focus success 1vely on the anomalles induced by the considered transition in the thermal expansion, the optical properties, the vibrational atomic spectrum, etc... In this view one will add terms in the Landau free-energy respectively corresponding to the mechantcal deformations, to the dielectric polarization, to other collective atomic displacements, etc...

The only rigid feature of the theory is its symmetry framework which imposes the form of the interactions between the various degrees of freedom, and the number of adjustable phenomenological coefficients. The form of the interactions determines the relationship between the laws governing the behaviour of the various physical quantities. The explanatory power of Landau's theory resides In the checking of the overall consistency of the observed laws.

Finally, an additional reason of consideration of tandau's theory is its specific status in respect to the statistical theory of critical phenomena (Wilson's theory). From this standpoint, Landau's theory appears as a necessary polnt of passage, and also as a tool. On the one hand, it is the order-parameter defined by Landau's theory whose fluctuations give rise to a singularity at the transition point. Accordingly, it is on this set of degrees of freedom that the statistical theory operates. On the other hand, Landau's theory provides the rules for constructing the effective Hamiltonian density, function of the orderparameter and of the secondary degrees of freedom, on which the renormallzation-group transfornations act. 
The contents of this book stems from three different objectives. In the first place, it is an introduction to the basic principles and techniques of Landau's theory, which is intended for teaching purposes. In this spirit, it includes an introduction to the peripheric group-theoretical and crystallographic concepts required to work out the theory. This part of the book is an expanded version of courses taught by the authors in varlous circumstances. Chapter $I$ is a self-contalned, simplified, introduction to the basic aspects of Landau's theory, which is weli adapted to a teaching at the undergraduate level. The first paragraphs of chapter II constitute a complete presentation of the theory. They involve a thorough discussion of the starting assumptions and an explicit decomposition of the steps of the argumentation. The same pedagogical purpose has presided over the writing of chapter III, of the two first paragraphs of chapter IV, and of the four first paragraphs of chapters VI and VII. These chapters are respectively devoted to the applications of Landau's theory to structural transitions, to f1rst-order transitions, and to magnet $1 \mathrm{c}$ and liquid-crystalline systems. Their contents is rooted in courses given at the graduate level.

A second purpose of the book is to provide the practical "rectpes" for applying Landau's theory to complex systems. In this view, each element of the method is fllustrated by several examples and the intermediate steps of many calculations are explicitely reproduced. Thus, one can find the constructions of the matrices of the order-parameter representation, or corepresentation (chaps. II, III, V and VI), the construction of the Landau free-energy (chaps. II and V), the description of the procedures of 1 ts minimization, and the method of identification of the low symmetry group for structural (chap. III) magnetic (chap. VI) and 1iquid crystal (chap. VI) systems. The procedure of application of the Landau criterion (chap. II) and of the Lifschitz one (chap. III) are exposed in deta1ls. For incommensurate systems (chap. V), an extensive description is given of the construction of the Lifschitz-invariant and other spatially dispersive terms, as well as the resolution of the equations relative to the standard situation of a two-component orderparameter. In the chapter devoted to liquid crystals, we have adopted a unified description of the various types of transitions occuring in these systems while existing theories often derive from a variety of approaches (chap. VII).

The last objective of the book is to Incorporate the developments which have arisen in the last 15 years from the extensive application of the theory to a varlety of physical systems. These developments involve several aspects. On the one hand, certain bases of the theory itself have been discussed. The meaning of the Lifschitz criterion has been analyzed by a number of authors and substantlally clarified through the study of incommensurate systems. Its initially derfvation by Lifschitz (chap. V) has been replaced by other derivations, physically more transparent, and mathematically more correct, though more complex (chap. III). Conversely, it has been understood that Lifschitz's derivation provided a method for the study of incommensurate systems (chap. V).

The second aspect of progress concerns the specification of the essential symmetrles underlying the Landau theory, through the replacement of groups acting in the physical space, by groups acting in the order 
parameter space. One has been able, by this means, to express in a more efficient way the intrinsic symmetry of the order-parameter, the symmetry of the truncated free-energy expansion, and the characteristics of the symmetry breaking across the transition. New procedures of minimization of the free-energy have been based on the consideration of these essential symmetries. These methods have clarifled the nature of the mathematical problem set by the Landau theory : find the absolute minimum of a $\mathrm{m}^{\text {th }}$ degree polynomial in several varlables, 1) having a local maximum at the origin, 1i) positive and infinite at infinity in any direction, iii) whose extrema have a symmetry-specifled degeneracy, and 1111) which possesses obligatory extrema along symmetry directions in the order-parameter space (chap. II, paragraph 4).

Finally, a large part of the book is devoted to the systems, already mentioned above, which have been studied, in recent years, by means of Landau's theory : continuous or discontinuous structural transitions involving coupling between several relevant degrees of freedom (chaps III and IV) magnetic transitions (chap. VI), transitions in liquidcrystals (chap. VII), incommensurate phases (chap. V). We have also outline the princlples of the application of the theory to icosahedral phases and to defects (chap. VIII).

In certain chapters, we had the cholce between various distinct approaches. It is worth pointing out that the theory of first-order transitions is essentially inspired by the works of Gufan and co-workers (chap. IV). The theories of magnetic and liquid crystal systems have respectively their roots in methods elaborated by Dzyaloshinski and by Indenbom and coworkers (chaps VI and VII).

The multiplicity of objectives pursued has the consequence that the different chapters or paragraphs are not treated evenly. In certain parts of the book, each statement is justified, while in others, dealing with more recently developped flelds, the reader is directed, for complete justification to appropriate reference works. The latter situation will be found, in particular, in large fractions of chapters IV-VIII.

In writing this book, we feel indebted to a number of colleagues. We are especially greatful to Louls Michel. From discussions with him we have learned most of the considerations pertaining to the essential symmetries of the Landau theory, which are Included in the book. We have also benefitted from meeting several times Yu $M$. Gufan and V.P. Dimitriev who have shared with us their deep understanding of the physical Implications of the Landau theory. The enlightening explanations of E. Brézin have been very helpful to clarify our view of the situation of Landau's theory from the standpoint of statistical physics. We had stimulating discussions with several experts in the handilng of Landau's theory, namely N. Boccara, V. Dvorak, and A.P. Levanyuk.

We are also indebted to F.W. Ainger, R. Chaves, A. Janner, T. Janssen J.Mozrzymas, P.M. Raccah, H. Schmid, and D. Weigel, who have invited us to teach the courses which have eventually resulted in this book. We acknowledge stimulating collaborations in various fields of application of Landau's theory with M. Clin, G. Errandonéa, J. Schneck, H. Schmid, P. Schobinger-Papamantelos, and R. Tekaia.

It is a pleasure to thank M. Coiret, P. Durand, and M.C. Mourier for their competent realization of the huge task of typing the manuscript. 


\section{THE LANDAU THEORY OF PHASE TRANSITIONS}


This page is intentionally left blank 


\section{TABLE OF CONTENTS} Chapter I : INTUITIVE APPROACH TO THE BASIC IDEAS OF LANDAU'S
THEORY

1 - INTRODUCTION

2 - MODEL EXAMPLE OF PHASE TRANSITION IN A CRYSTAL 2

2.1 - The system and its degrees of freedom 2

2.2 - Symmetry of the two phases 3

2.3 - Variational free-energy associated to the system 4

2.4 - Symmetry properties of $F$ and form of its $2^{\text {nd }}$ degree
Taylor expansion

2.5 - Decoupling of the $\left(\mathrm{p}_{x}, \mathrm{p}_{y}\right)$ and $\mathrm{p}_{z}$ degrees of freedom

2.6 - Need for an expansion of degree higher than 2 , below

2.7 - Simple physical consequences of the $4^{\text {th }}$ degree order-
parameter expansion

2.8 - Symmetry considerations 15

2.9 - Secondary order-parameters 19

3 - CONCLUSIONS 23

Chapter II : FORMULATION OF LANDAU'S THEORY

1 - INTRODUCTION 25

2 - BASIC CONCEPTS OF GROUP REPRESENTATIONS 25

2.1 - Irreducible representations of a group $G_{0} \quad 26$

2.2 - Group theoretical properties used in the
formulation of Landau's theory

3 - LANDAU'S THEORY

3.1 - Variational and equilibrium particle density and
free-energy

3.2 - Decomposition of the density increment into
irreducible parts

3.3 - Scalar variational degrees of freedom 38

3.4 - Second degree Taylor expansion of $F\left(n_{k r}\right)$

3.5 - Definition of the order-parameter $\mathrm{kr} \quad 40$

3.6 - Order-parameter expansion and nature of the symmetry
change

4 - TECHNICAL GROUP THEORETICAL ASPECTS OF LANDAU'S THEORY 47

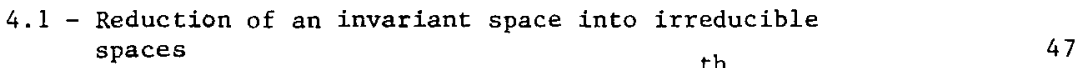

4.2 - Tensorial products of invariant spaces $n^{\text {th }}$ power of
a representation

4.3 - Symetrized $n^{\text {th }}$ power of a representation 49 
4.4 - The Landau Condition

4.5 - Invariant polynomials and image of $G_{0}$ in the OP-representation

4.6 - Minimization of the free-energy and symmetry breaking

5 - SECONDARY ORDER-PARAMETER

5.1 - Coupling between secondary and primary OP

5.2 - Possible symmetries of secondary $O P$, and form of $\emptyset\left(\zeta_{s}, \eta_{r}\right)$

5.3 - Equilibriun value of the secondary op nearby $T_{c}$

5.4 - Irrelevance of the secondary OP, to the symmetry below $\mathrm{T}_{\mathrm{C}}$

6 - CONCLUSIONS

\section{Chapter III : CONTINUOUS STRUCTURAL TRANSITIONS BETWEEN} PERIODIC PHASES

1 - INTRODUCTION

2 - CRYSTALLOGRAPHIC SPACE-GROUPS AND THEIR REPRESENTATIONS

2.1 - Crystallographic space-groups

2.2 - Irreducible representations of the space-groups

3 - THE LIFSCHITZ CRITERION

3.1 - Derivation of the Lifschitz criterion

3.2 - Procedure of application of the Lifschitz criterion

3.3 - Selection of $\vec{k}_{0}$-vectors resulting from the Litschitz criterion

3.4 - Lifschitz criterion and periodicity of the lowsymmetry phases

4 - IMAGES AND FREE-ENERGIES FOR ACTIVE REPRESENTATIONS

4.1 - Active representations of a space-group

4.2 - Images of $G_{0}$ for active representations

4.3 - Free-energies for active representations

5 - DETERMINATION OF THE LOW-SYMMETRY SPACE GROUP G

6.1 - Ferroicity and point-symmetry change at $T_{C}$

6.2 - Proper and improper ferroics

7 - EXAMPLE OF THE STRUCTURAL TRANSITION IN GADOLINIUM MOLYBDATE 
Chapter IV : FIRST ORDER TRANSITIONS

1 - INTRODUCTION

2 - FIRST ORDER TRANSITIONS ASSOCIATED WITH HIGH-DEGREE EXPANS IONS OF THE ORDER-PARAMETER

2.1 - Case with $n=1$ and $d=6$ : general features of firstorder transitions

2.2 - General features of phase diagrams with onecomponent order-parameter expansions

2.3 - Phase diagrams with two-component order-parameters

2.4 - Phase diagrams with multi-component orderparameters

2.5 - Experimental examples

3 - TRANSITIONS PREDICTED TO BE DISCONTINUOUS BY THE LANDAU CONDITION

3.1 - Role of third order invariants of the orderparameter in the phenomenological description of structural transitions

3.2 - Experimental examples

4 - TRANSITIONS PREDICTED TO BE DISCONTINUOUS BY THE LIFSCHITZ CONDITION

5 - TRANSITIONS ASSOCIATED WITH MORE THAN ONE ORDER-PARAMETER

5.1 - General properties of phase transitions associated with several order-parameters

5.2 - Examples of phase diagrams associated with two order-parameters

6 - PHENOMENOLOGICAL THEORY OF PHASE TRANSITIONS WHICH HAVE NO GROUP-SUBGROUP RELATIONSHIP BETWEEN THE PHASES

6.1 - Reconstructive transitions for which the orderparameter is a sinusoidal function of the atomic shifts

6.2 - Reconstructive transitions for which the variational parameter is the degree of occupation of a latent unit cell 
Chapter V : IANDAU THEORY OF INCOMMENSURATE PHASES

1 - INTRODUCTION

1.1 - Standard experimental scheme for INC systems

1.2 - Basic ideas for the adaptation of Landau's theory

2 - SYMMETRY PROPERTIES OF THE ORDER-PARAMETER AND OF THE FREE-ENERGY

2.1 - Order-parameter and free-energy for an incommensurate wavevector

2.2 - order-parameter and free-energy for the lock-in vector $\vec{k}_{L}$

2.3 - Free-energy density for a modulated orderparameter; Lifschitz invariant

2.4 - Three standard examples

3 - QUALITATIVE INTERPRETATION OF THE EXPERIMENTAL SITUATION

3.1 - Simplified formulation of the first theoretical scheme

3.2 - Refinement of the first theoretical scheme

3.3 - Second theoretical scheme, in the presence of a Lifschitz invariant

4 - PHENOMENOLOGICAL THEORY FOR A TWO-COMPONENT ORDERPARAMETER IN THE PMA APPROXIMATION

4.1 - The 8 -equation

4.2 - The sinusoidal limit $\left(\mu^{2} \approx 0 ; \mathrm{T}<\mathrm{T}_{\mathrm{I}}\right) \quad 269$

4.3 - General form of the free energy for $\mu^{2} \neq 0 \quad 270$

4.4 - Multisoliton 1imit $\left(\mu^{2} \approx 1 ; \mathrm{T} \approx \mathrm{T}_{\mathrm{L}}\right)$

4.5 - Macroscopic quantities and anomalies of the susceptibilities

5 - EXTENSIONS

5.1 - Lifting of the PMA

5.2 - Phenomenological theory in the absence of a Lifschitz invariant

5.3 - Effect of additional spatially dispersive terms

5.4 - Higher number of order-parameter components and of modulation directions

6 - CONCLUSTONS

Chapter VI : THE LANDAU THEORY OF MAGNETIC TRANSITIONS

1 - INTRODUCTION

2.2 - Identification of the type of magnetic ordering associated with a given magnetic group 
2.3 - Magnetic lattices and magnetic space-groups 312

3 - IRREDUCIBLE COREPRESENTATIONS OF THE MAGNETIC GROUPS 313

4 - SPECIFIC FORMULATION OF THE LANDAU THEORY FOR MAGNETIC SYSTEMS AND EXCHANGE SYMMETRIES

4.1 - Formulation of the Landau theory 315

$\begin{array}{ll}4.2 \text { - Exchange symmetry } & 317\end{array}$

5 - PRACTICAL APPLICATION OF THE LANDAU THEORY TO MAGNETIC
TRANSITIONS : SOME EXAMPLES

5.1 - Introduction 321

5.2 - Phase transitions from the paramagnetic group
Pca2 $11^{\prime}$ at $k=0$

5.3 - Phase transitions in $\alpha-\mathrm{Fe}_{2} \mathrm{O}_{3} \quad 329$

5.4 - Latent antiferromagnetism in nickel-iodine boracite 341

6 - APPLICABILITY OF THE LANDAU THEORY TO MAGNETIC SYSTEMS : ORDER PARAMETER SYMMETRIES, FIRST-ORDER TRANSITIONS AND INCOMMENSURATE MAGNETIC PHASES

6.1 - Order-parameter symmetries for second and firstorder transitions in magnetic systems

6.2 - Specific features of the Landau theory of incommensurate magnetic systems

7 - COUPLING OF THE MAGNETIC ORDER-PARAMETER TO NON-MAGNETIC PHYSICAL QUANTITIES : SPONTANEOUS MAGNETOSTRUCTURAL EFFECTS

7.1 - Introduction

7.2 - Spontaneous magnetostriction 362

7.3 - Spontaneous piezomagnetism 365

7.4 - Spontaneous magnetoelectricity 367

7.5 - Coupling between structural and magnetic transitions 369

Chapter VII : THE LANDAU THEORY OF LIQUID CRYSTALS

1 - INTRODUCTION

2 - SYMMETRY GROUPS OF LIQUID-CRYSTALS $\quad 374$

2.1 - An introductory example 374

2.2 - The macroscopic space-groups of liquid crystals 377

3 - THE IRREDUCIBLE REPRESENTATIONS OF LIQUID CRYSTAL GROUPS 379

3.1 - Irreducible representations of the G point-groups 379

3.2 - Irreducible representations of the space-groups S 383

4 - PROBABILITY DENSITIES, ORDER-PARAMETERS AND THERMODYNAMIC
POTENTIALS FOR LIQUID CRYSTAL TRANSITIONS

4.1 - Probability densities 388

4.2 - Primary and secondary order-parameters 389 
xviii

4.3 - Thermodynamic potentials

390

4.4 - Practical procedure for the determination of the symmetry changes which take place at Liquid Crystal Transitions

5 - APPLICABILITY OF THE LANDAU THEORY TO PHASE TRANSITIONS IN LIQUID-CRYSTALS

5.1 - The nematic to smectic A transition

5.2 - The nematic-smectic A-smectic C phase diagram

5.3 - Reentrant nematic phases

407

5.4 - The uniaxial to biaxial nematic transition

5.5 - Transitions between smectic phases

6 - MODULATED LIQUID CRYSTAL PHASES

7 - TILTING ORDER AND BOND-ORIENTATIONAL ORDER IN SMECTIC PHASES

8 - FERROELECTRIC, FLEXOELECTRIC AND PIEZOELECTRIC EFFECTS

9 - TRANSIIIONS FROM THE ISOTROPIC PHASE

Chapter VIII : RECENT DEVELOPMENTS AND FIELD OF VALIDITY OF LANDAU'S THEORY

1 - INTRODUCTION

2 - STABILITY OF ICOSAHEDRAL QUASI-CRYSTALLINE PHLASES

2.1 - Landau's model of the liquid-solid phase transition

2.2 - Alexander and MC Tague's extension of the model

2.3 - Application of the model to icosahedral phase

3 - INFLUENCE OF DEFECTS ON PHASE TRANSITIONS

3.1 - Classification of defects

3.2 - Phenomenological theory for symmetry-breaking point defects

4.1 - Validity of the theory and statistical fluctuations

4.2 - Preservation of the validity of the symmetry aspects of Landau's theory

4.3 - Cases in which the thermodynamical results of Landau's theory are preserved

4.4 - Behaviour of physical quantities in the critical range 